\title{
Jefferson Lab phenomenology: an overview
}

\author{
W. Melnitchouk ${ }^{a}$ \\ ${ }^{a}$ Jefferson Lab \\ 12000 Jefferson Avenue \\ Newport News, VA 23606, USA
}

Experiments at Jefferson Lab are pushing the frontiers of our knowledge about the structure and dynamics of nucleons and nuclei. I will review a selection of recent results and discuss their impact on our understanding of hadron structure.

\section{INTRODUCTION}

The Thomas Jefferson National Accelerator Facility (Jefferson Lab) is a $6 \mathrm{GeV}$ electron accelerator located in Newport News, Virginia, USA. Since the experimental program began in 1996, Jefferson Lab has developed into the premier electron accelerator facility in the world for precision studies of the structure of the atomic nucleus at the quark level. The Continuous Electron Beam Accelerator Facility (CEBAF) uses superconducting radio-frequency technology to produce an electron beam of unprecedented high luminosity (up to $10^{39} \mathrm{~cm}^{-1} \mathrm{~s}^{-1}$ ) and polarization. The electron beam can be split for use in simultaneous experiments in three experimental halls (Halls A, B and C).

The high-precision data from recent Jefferson Lab experiments are providing stringent tests of theories of hadronic structure, and in some cases forcing re-evaluations of commonly accepted wisdom. It is clearly impossible to review in 8 pages all of the exciting developments which have taken place at Jefferson Lab over the past 8 years, so I will select a few recent highlights and discuss their impact on our understanding of the structure of hadrons in QCD. I will focus on three main areas: baryon spectroscopy, elastic proton form factors, and inclusive structure functions.

One of the most exciting developments in hadronic physics for a long time has been the reported discovery of the $\Theta^{+}$pentaquark. I discuss the current status of pentaquark searches, as well as other results from excited nucleon spectroscopy. The study of exclusive reactions has provided some unexpected results for electromagnetic form factors of the proton. As a byproduct, it has led to a re-evaluation of the validity of the one-photon exchange approximation in electron-nucleon scattering. In inclusive scattering, I review the spectacular observations of quark-hadron duality in inclusive structure functions measured in the nucleon resonance and deep inelastic regions. Finally, I preview future plans for CEBAF at $12 \mathrm{GeV}$, and the prospects of a high-luminosity polarized electron-ion collider.

\section{BARYON SPECTROSCOPY}

All of the known baryons observed in Nature have quantum numbers which can be constructed from 3 valence quarks. Baryons whose quantum numbers require a minimum valence quark content of $q q q q \bar{q}$ are therefore considered "exotic". A number of experiments have reported observations of these "pentaquarks" in the past $\sim 18$ months (for recent reviews see Refs. [1,2]).

The first observation of the strangeness $S=$ +1 , charge +1 baryon, referred to as the $\Theta^{+}$, was reported by the LEPS Collaboration at the SPring-8 facility in Japan [3] in the inclusive reaction $\gamma n \rightarrow K^{-}\left(K^{+} X\right)$. Here the target neutron was bound in a carbon nucleus, and the residual nucleus was assumed to be a spectator. The minimal quark content to produce such a state is $u u d d \bar{s}$. The mass of the $\Theta^{+}$was found to be 
around $1.54 \mathrm{GeV}$ with a width less than $25 \mathrm{MeV}$.

This observation was confirmed shortly thereafter by the CLAS Collaboration at Jefferson Lab in photoproduction experiments on deuterium [4] and hydrogen [5]. A number of other collaborations also reported observations of the $\Theta^{+}$baryon at similar masses, and with widths less than a few $\mathrm{MeV}$, for a total of around 11 positive sightings. In addition, evidence for the strangeness $S=-2$ exotic state $\Xi_{5}^{--}$, with quark content $d d s s \bar{u}$, has been claimed by the NA49 Collaboration at CERN [6]. Figure 1 shows the result from the deuterium measurement by CLAS [4].

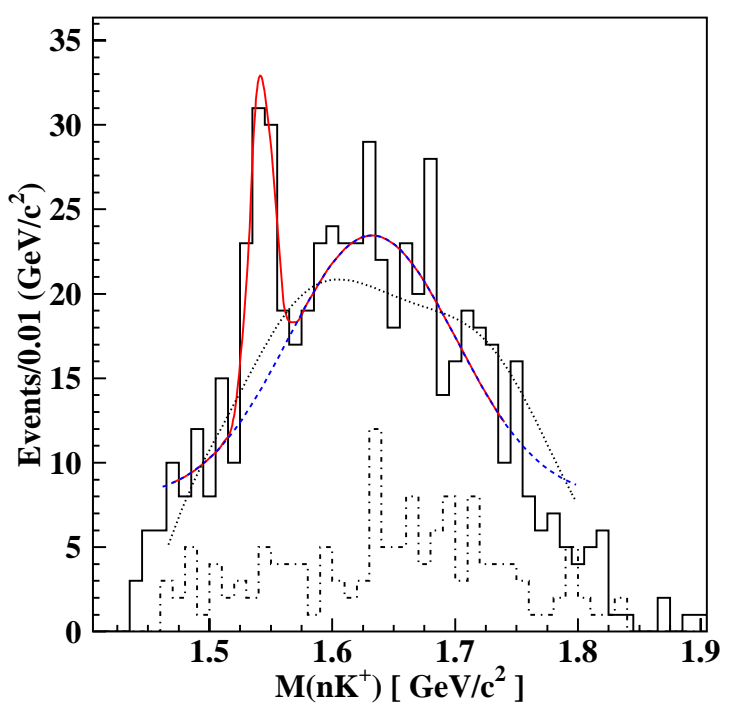

Figure 1. Invariant mass of the $n K^{+}$system, with strangeness $S=+1$, from Ref. [4], showing a sharp peak at a mass $1.542 \mathrm{GeV}$. The solid line is a fit to the peak on top of the smooth background (dashed line). The dotted curve is the simulated background, and the dot-dashed histogram shows the spectrum of events associated with $\Lambda(1520)$ production.

Needless to say, these reports have stimulated tremendous excitement in the community, and a plethora of theoretical papers trying to explain the features of these states. One of the biggest puzzles has been the unusually narrow width, which most experiments have only been able to put upper limits on (basically, limited by the experimental resolution). Indeed, reanalyses of old $K N$ scattering data [7] revealed that a width greater than about $1 \mathrm{MeV}$ would be in conflict with the $K N$ cross sections and phase shifts. It is very difficult to accommodate such a small width within any reasonable theoretical framework or model.

Moreover, because the background on top of which the $\Theta^{+}$signal sits is fitted rather than calculated, there have been concerns raised that the reported signal may be due to kinematical reflections [8]. Currently the experimental situation is still unresolved. Along with the 10 or so positive observations, there have been about as many null results. Most of these have been in inclusive highenergy reactions, which have seen no evidence for either the $S=+1 \Theta^{+}$state nor the $S=-2 \Xi_{5}^{--}$ exotic. If the pentaquarks do exist, it appears that their production mechanism may be exotic, along with their quantum numbers.

New data from Jefferson Lab, as well as SPring8 , with several times the statistics of the earlier experiments, are currently being analyzed [2], and new experiments are planned at other facilities. We can hope that by the time these proceedings are published, a definitive statement can be made about the existence of the $\Theta^{+}$. If the results are positive, further progress towards determining its spin and parity quantum numbers, as well as its mass and width, will be priorities.

Before leaving spectroscopy, we should note that even though considerable attention has been devoted to the pentaquarks searches, other more "mainstream" areas of baryon spectroscopy are also being actively studied at Jefferson Lab. An example is the recent evidence from CLAS for a possible new $P_{13}$ excited nucleon state with mass $\sim 1700 \mathrm{MeV}$ [9], which does not appear to be compatible with the properties of the known $N^{*}$ states in this mass region. The conclusion of these studies is that either the couplings of the known $P_{13}(1720)$ state need to be drastically changed 
from those given in the Particle Data Book, or a new $P_{13}$ state must be introduced.

\section{PROTON FORM FACTORS}

Electromagnetic form factors are the most fundamental observables which characterize the composite nature of the nucleon. Several decades of elastic form factor experiments with electron beams, including recent high-precision measurements at Jefferson Lab and elsewhere, has provided considerable insight into the detailed structure of the nucleon.

In the usual one-photon exchange approximation, the electromagnetic current operator is parameterized in terms of the Dirac $\left(F_{1}\right)$ and Pauli $\left(F_{2}\right)$ form factors,

$\Gamma_{(1 \gamma)}^{\mu}=F_{1}(q) \gamma^{\mu}+\frac{i \sigma^{\mu \nu} q_{\nu}}{2 M} F_{2}(q)$,

where $q$ is the momentum transfer to the nucleon, and $M$ is the nucleon mass. The resulting cross section depends on two kinematic variables, conventionally taken to be $Q^{2} \equiv-q^{2}$ and either the scattering angle $\theta$ or the virtual photon polarization $\epsilon=\left(1+2(1+\tau) \tan ^{2}(\theta / 2)\right)^{-1}$, where $\tau \equiv Q^{2} / 4 M^{2}$. In terms of the Sachs electric and magnetic form factors,

$$
\begin{aligned}
G_{E}\left(Q^{2}\right) & =F_{1}\left(Q^{2}\right)-\tau F_{2}\left(Q^{2}\right), \\
G_{M}\left(Q^{2}\right) & =F_{1}\left(Q^{2}\right)+F_{2}\left(Q^{2}\right),
\end{aligned}
$$

the (reduced) Born cross section can be written

$\sigma_{R}=G_{M}^{2}\left(Q^{2}\right)+\frac{\epsilon}{\tau} G_{E}^{2}\left(Q^{2}\right)$.

\subsection{Extraction methods}

The standard method which has been used to determine the electric and magnetic form factors has been the Rosenbluth, or longitudinaltransverse (LT), separation method. Because the form factors in Eq. (4) are functions of $Q^{2}$ only, studying the cross section as a function of the polarization $\epsilon$ at fixed $Q^{2}$ allows one to extract $G_{M}^{2}$ from the $\epsilon$-intercept, and the ratio $G_{E} / G_{M}$ from the slope in $\epsilon$. The results of the Rosenbluth measurements for the proton have generally been consistent with $\mu_{p} G_{E}^{p} / G_{M}^{p} \approx 1$ for $Q^{2}<6 \mathrm{GeV}^{2}$ [10-12], where $\mu_{p}$ is the nucleon magnetic moment, as Fig. 2 illustrates.

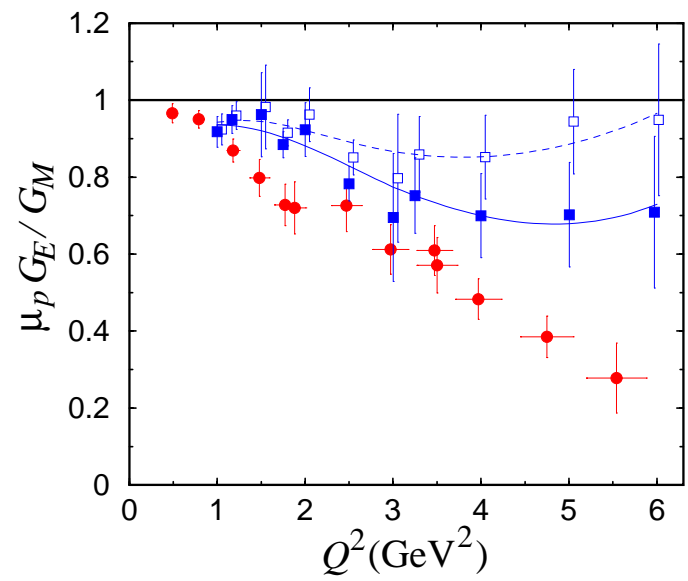

Figure 2. The ratio of form factors measured using the LT separation (open squares) and polarization transfer [13] (circles) techniques, together with the global fit (dashed line) to the LT data [10]. The unshifted LT data represent a binned average of all LT separated data points with normalization factors determined by the global fit in Ref. [10]. Filled squares show the shift in the LT results due to the two-photon-exchange corrections (offset for clarity), and the solid line shows the effect on the global fit. (From Ref. [14].)

An alternative method of extracting the ratio $R$ was used more recently at Jefferson Lab [13], in which a polarized electron beam scatters from an unpolarized target, and the polarization of the recoiling proton is detected. From the ratio of the transverse to longitudinal recoil polarizations one finds

$\frac{G_{E}}{G_{M}}=-\frac{P_{t}}{P_{l}} \frac{E_{i}+E_{f}}{2 M} \tan \frac{\theta}{2}$,

where $E_{i}\left(E_{f}\right)$ is the initial (final) electron energy, and $P_{t}\left(P_{l}\right)$ is the polarization of the recoil proton transverse (longitudinal) to the proton momentum in the scattering plane. The polarization transfer experiments yielded strikingly different results compared with the Rosenbluth over the same range in $Q^{2}$ [10], as illustrated by the filled circles in Fig. 2. Recall that in perturbative QCD one expects $F_{1} \sim Q^{2} F_{2}$, or $G_{E} \sim G_{M}$, at large 
$Q^{2}$, so that these results imply a strong violation of perturbative scaling behavior.

\subsection{Two-photon exchange}

Attempts to reconcile the LT and polarization transfer measurements have been made by several authors $[15,14,16]$, who have investigated the possibility that $2 \gamma$-exchange effects, which form part of the radiative corrections, may account for at least part of the observed discrepancy. Although the overall magnitude of the $2 \gamma$-exchange contribution is suppressed by order $\alpha$ relative to the Born term, in practice a change in the $\epsilon$ slope of $5-6 \%$ is all it takes to bring the two measurements into agreement [17].

One should note that part of the $2 \gamma$-exchange contributions (the "box" and "crossed box" diagrams) are included in the standard radiative correction treatments [18], albeit in an approximate manner. Namely, one usually employs the soft-photon approximation, in which one of the two photons in the internal loop is placed on its mass shell. This makes the contribution factorizable into a product of the Born term and an overall multiplicative factor. In effect it neglects the structure of the nucleon. A partial attempt to go beyond the soft-photon approximation was made in Ref. [19], and a full calculation taking the finite size of the nucleon into account was made by Blunden et al. [14].

While the soft-photon approximation preserves the linearity of the $\epsilon$ plot, allowing one to extract $G_{E} / G_{M}$ from the linear slope in $\epsilon$, the full calculation of the $2 \gamma$ contribution including effects of nucleon structure in principle gives rise to nonlinearity in $\epsilon$. To estimate the influence of the $2 \gamma$ exchange corrections on the electric to magnetic form factor ratio, Blunden et al. approximate the full $\epsilon$ dependence by a linear function over a limited range of $\epsilon$, where the non-linearity is not large. This approximation becomes progressively worse with increasing $Q^{2}$, but is reasonable up to $Q^{2} \sim$ few $\mathrm{GeV}^{2}$. In the actual data analyses one should apply the full $2 \gamma$ correction directly to the data.

The effect of the $2 \gamma$-exchange terms on $R$ is illustrated in Fig. 2 by the solid squares (and the solid curve), which are shifted by the $2 \gamma$ correc- tion. The $2 \gamma$ corrections are significant, and have the proper sign and magnitude to resolve a large part of the discrepancy between the two experimental techniques.

New experiments at Jefferson Lab will measure the $\epsilon$ dependence of the cross section to look for deviations from linearity in $\epsilon$, and also measure the individual polarizations $P_{t}$ and $P_{l}$ in Eq. (5) instead of only the ratio. Finally, a new experiment will simultaneously scatter electrons and positrons elastically from a proton target [20]. Since the interference of the $2 \gamma$-exchange and Born diagrams changes sign for electrons and positrons, whereas the Born term does not, this will unambiguously determine the size of the $2 \gamma$ contribution.

\section{STRUCTURE FUNCTIONS}

Elastic form factors provide information about the spatial distributions of quarks in the nucleon. Structure functions, on the other hand, which are measured in inclusive deep inelastic scattering of electrons from nucleons, determine the (lightcone) momentum distributions of quark and gluons (or generically partons) in the nucleon, in terms of the light-cone momentum fraction $x$ of the nucleon carried by the parton. Recent experiments at Jefferson Lab have provided a number of interesting results in the study of parton distributions and structure functions.

\subsection{Valence quarks at large $x$}

One of the most fundamental properties of the nucleon is the structure of its valence quark distributions. Knowledge of the valence quark structure of the nucleon is vital for instance for understanding the mechanisms by which spin-flavor symmetry is broken at large $x$. From another perspective, quark distributions at large $x$ are also essential for determining high-energy cross sections at collider energies, such as in searches for the Higgs boson or for new physics beyond the Standard Model, where structure information at $x \sim 0.6-0.8$ feeds down to lower $x$ at higher values of $Q^{2}$ through perturbative $Q^{2}$ evolution.

Most of the experimental studies of nucleon structure over the last decade have been focused 
on the small- $x$ region populated mainly by sea quarks. The valence quark structure at large $x$ is in contrast relatively poorly known, due to the rapidly decreasing cross sections as $x \rightarrow 1$. As a result, answers to such basic questions as why the $d$ quark distribution at large $x$ appears to be smaller (or "softer") than that of the $u$ remain unresolved.

The need for reliable large- $x$ data is even more pressing for the spin-dependent quark distributions. Spin degrees of freedom allow access to information about the structure of hadrons not available through unpolarized processes. Spin-dependent quark distributions are usually extracted from measurements of the spinpolarization asymmetry, $A_{1}$, which is approximately given by the ratio of spin-dependent to spin-averaged structure functions:

$A_{1}(x) \approx \frac{g_{1}(x)}{F_{1}(x)}$,

where, to leading order,

$$
\begin{aligned}
& F_{1}(x)=\frac{1}{2} \sum_{q} e_{q}^{2}(q(x)+\bar{q}(x)), \\
& g_{1}(x)=\frac{1}{2} \sum_{q} e_{q}^{2}(\Delta q(x)+\Delta \bar{q}(x)) .
\end{aligned}
$$

Here $q$ and $\Delta q$ are defined as the sum and difference, respectively, between quark distributions with spin aligned and anti-aligned with the spin of the nucleon, $q=q^{\uparrow}+q^{\downarrow}$ and $\Delta q=q^{\uparrow}-q^{\downarrow}$.

Various models exist which make quite specific predictions for the behavior of quark distributions at large $x$. Some of these can be related to phenomena such as the hyperfine splitting of the baryon and meson mass spectra [21]. A quark hyperfine interaction, such as that due to one-gluon exchange, or pion exchange (which can induce a higher energy for the $S=1$ spectator "diquark" in the nucleon wave function) will necessarily give rise to a larger mass for the $\Delta$ isobar since the quark wave function for the $\Delta$ has all "diquark" configurations with $S=1$. If the $S=0$ states are dominant at large $x$, the $d$ quark distribution will be suppressed relative to that of the $u$. This mechanism also leads to specific predictions for the polarization asymmetries as $x$ becomes large and close to unity. Namely, the polarization asymmetry $A_{1}^{n}$ should approach unity in the limit $x \rightarrow 1$, while the ratios of the polarized to unpolarized distributions for individual flavors behave as $\Delta u / u \rightarrow 1$ and $\Delta d / d \rightarrow-1 / 3$ as $x \rightarrow 1$.

Arguments based on hadron helicity conservation within perturbative QCD, on the other hand, predict that the dominant components of the proton valence wave function at large $x$ are those associated with states in which the total "diquark" helicity, or spin projection, $S_{z}$, is zero [22]. Consequently, scattering from a quark polarized in the opposite direction to the proton polarization is suppressed relative to the helicity-aligned configuration. This leads to a faster approach to unity for $A_{1}^{n}$, since with this mechanism both $\Delta u / u \rightarrow 1$ and $\Delta d / d \rightarrow 1$ as $x \rightarrow 1$.

From Eqs. (6)-(8) the ratios $\Delta u / u$ and $\Delta d / d$ can be reconstructed from the polarization asymmetries $A_{1}^{p}$ and $A_{1}^{n}$, and knowledge of the unpolarized $d / u$ ratio. While there are data for $A_{1}^{p}$ which extend to $x \sim 0.7$, until recently essentially no data at all existed on $A_{1}^{n}$ for $x \geq 0.4$. Recently measurements in Hall A at Jefferson Lab on polarized ${ }^{3} \mathrm{He}$ targets have provided for the first time accurate data on $A_{1}^{n}$, allowing the decomposition of the spin and flavor ratios to be made.

The new data show a clear trend that $A_{1}^{n}$ becomes positive at large $x$ [23]. Combined with the world proton data on $A_{1}^{p}$, the ratios $\Delta u / u$ and $\Delta d / d$ are shown in Fig. 3. The results agree with the perturbative QCD fits to the previous data, and the trend agrees with the hyperfineperturbed relativistic quark models predictions. On the other hand, the new data do not agree with the prediction from $\mathrm{pQCD}$-based hadron helicity conservation. This suggests that effects such as the quark orbital angular momentum may play an important role in this kinematic region. Extension of precision measurements of $A_{1}^{n}$ to higher $x$ and wider $Q^{2}$ range is planned with the future Jefferson Lab $12 \mathrm{GeV}$ energy upgrade (see Sec. 5 below).

\subsection{Duality in structure functions}

At large $x$ the kinematics of inclusive electron scattering implies that, for fixed $Q^{2}$, one probes deeper into the resonance region at lower final 


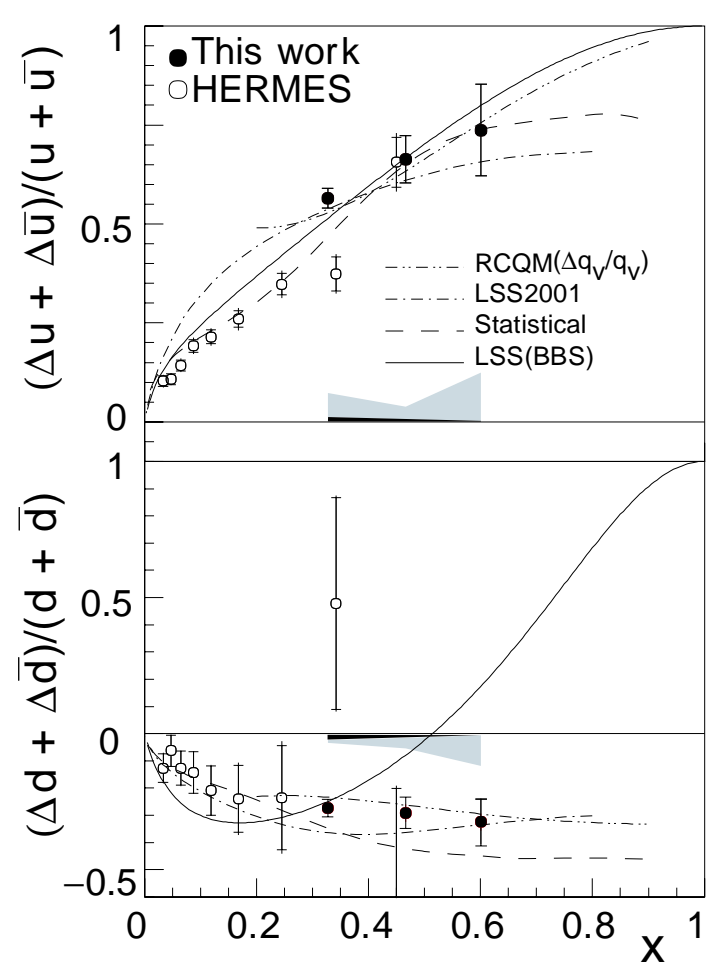

Figure 3. Ratios of polarized to unpolarized $u$ and $d$ quark distributions in the proton from Ref. [23] (filled circles) compared with earlier HERMES data (open circles) and various models and parameterizations.

state hadron masses $W, x=Q^{2} /\left(Q^{2}+W^{2}-M^{2}\right)$. Of course the elastic limit where $W=M$ corresponds to $x=1$. As one goes from the deep inelastic region where the structure functions are smoothly varying functions of $x$, and weakly dependent (approximately scaling) functions of $Q^{2}$, to lower $W$ (higher $x$ ), one encounters the region where oscillations in the structure function data become prominent. The oscillations are associated with the production of nucleon resonances, which appear as peaks and troughs corresponding to the various resonances.

One of the most interesting results to come from recent Jefferson Lab experiments has been the observation that the structure functions mea- sured in the resonance region are on average approximately equivalent to the scaling function which describes the high-energy data. This equality between the resonance region (characterized by hadronic bound states) and the deep inelastic continuum (characterized by scattering from free quarks) is referred to as "quark-hadron duality". First observed by Bloom and Gilman some 3 decades ago [25], this duality has been spectacularly confirmed by the new high-precision Jefferson Lab data on the proton $F_{2}^{p}$ structure functions. As illustrated in Fig. 4, the data clearly demonstrate that duality works remarkably well for each of the low-lying resonances to rather low values of $Q^{2}\left(\sim 0.5 \mathrm{GeV}^{2}\right)$. The $F_{2}^{p}$ structure function here is shown as a function of the Nachtmann scaling variable $\xi=2 x /(1+$ $\left.\sqrt{1+4 M^{2} x^{2} / Q^{2}}\right)$, which takes into account kinematical target mass corrections.

Before the advent of QCD, the quark-hadron duality in structure functions was interpreted in the context of finite-energy sum rules, in analogy with the $s$ - and $t$-channel duality observed in hadron-hadron scattering. In QCD, this duality can be reformulated in the language of the operator product expansion, in which moments of structure functions are organized in powers of $1 / Q^{2}$. For the $F_{2}$ structure function, for example, one has for the $n$-th moment

$$
\begin{aligned}
M_{2}^{(n)}\left(Q^{2}\right) & =\int_{0}^{1} d x x^{n-2} F_{2}\left(x, Q^{2}\right) \\
& =\sum_{\tau=2}^{\infty} \frac{A_{\tau}^{(n)}\left(\alpha_{s}\left(Q^{2}\right)\right)}{Q^{\tau-2}},
\end{aligned}
$$

where $A_{\tau}^{(n)}$ are the matrix elements of operators with twist $\leq \tau$ (where twist is defined as the mass dimension minus the spin, $n$, of the operator).

The leading terms in Eq. (10) are associated with free quark scattering, and are responsible for the scaling, while the $1 / Q^{2}$ terms involve interactions between quarks and gluons. The weak $Q^{2}$ dependence of the low moments of the structure function is then interpreted as indicating that the non-leading, $1 / Q^{2}$-suppressed, interaction terms do not play a major role even at low $Q^{2}$.

An important consequence of duality is that the strict distinction between the resonance and 


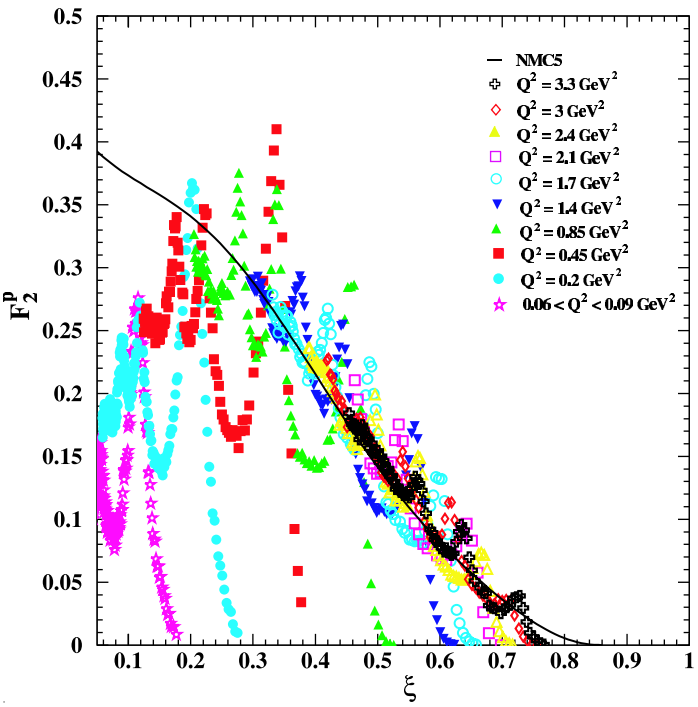

Figure 4. Proton $F_{2}^{p}$ structure function data from Jefferson Lab and SLAC in the resonance region in the range $0.06<Q^{2}<3.30 \mathrm{GeV}^{2}$, as a function of the variable $\xi$ [24]. The solid curve is a fit to deep inelastic data at the same $\xi$ but higher $\left(W^{2}, Q^{2}\right)$, shown here at $Q^{2}=5 \mathrm{GeV}^{2}$.

deep inelastic regions becomes entirely artificial. To illustrate this, consider that at $Q^{2}=1 \mathrm{GeV}^{2}$ around $2 / 3$ of the total cross section comes from the resonance region, $W<2 \mathrm{GeV}$. However, the resonances and the deep inelastic continuum conspire to produce only about a $10 \%$ higher-twist correction to the lowest moment of the scaling $F_{2}$ structure function at the same $Q^{2}$. Even though each resonance is built up from a multitude of twists, when combined the resonances interfere in such a way that they resemble the leading-twist component!

This by itself is quite a remarkable observation. But how can it be made useful in practice? If the degree to which duality holds, or the extent to which duality is violated, is understood, then the resonance data, when properly averaged, can be used to extract information on the leading-twist (scaling) parts of structure functions. Furthermore, if the inclusive-exclusive connection via lo- cal duality is taken seriously, one can relate structure functions measured in the resonance region to electromagnetic transition form factors.

Conversely, structure function data, and in particular the moments, at intermediate $Q^{2}$ values can be used to extract matrix elements of higher-twist operators. Recent analyses of spindependent structure functions of the proton and neutron have allowed the determination of quantities such as the color-electric and magnetic polarizabilities, which describe the response of the color gluon fields in the nucleon to the spin of the nucleon [26-28]. Although these have been studied to a much lesser extent than the scaling contributions, the higher-twist matrix elements describe long-range, non-perturbative correlations between partons in the nucleon, and as such are as fundamental properties of the nucleon as the spin and momentum carried by quarks.

\section{FUTURE PLANS}

The above examples have only touched the surface of the exciting new results that have been coming from Jefferson Lab in recent years. Programs to measure the strangeness content of the nucleon, to map out few-body form factors at high $Q^{2}$, to investigate the quark-hadron transition in exclusive meson-production, as well as new experiments to explore the unchartered territory of generalized parton distributions in deeply virtual Compton scattering, will undoubtedly provide valuable information on the quark structure of hadrons and nuclei in the years to come.

Beyond the next 6-7 years, the recent formal approval of the $12 \mathrm{GeV}$ energy upgrade of the CEBAF accelerator means that the next phase of Jefferson Lab's mission can get under way [29]. The centerpiece of the $12 \mathrm{GeV}$ upgrade will be the construction of a new experimental hall (Hall D), which will enable a systematic search for mesons with exotic quantum numbers, i.e., $J^{P C}$ values which cannot be constructed out of $q \bar{q}$ pairs alone, but which require dynamical glue in the wave function. In addition, the $12 \mathrm{GeV}$ upgrade will permit measurement of deep inelastic structure functions at large values of $x$, beyond anything which has been achievable at laboratories else- 
where, as well as more thorough exploration of the generalized parton distributions.

The future beyond the $12 \mathrm{GeV}$ upgrade may involve a high-luminosity electron-ion collider [30]. This collider - dubbed "ELIC" (Electron-Light Ion Collider) - would require the construction of a new proton ring, into which the electron beam from CEBAF would be injected. One novel element here would be the possibility of operating ELIC as both a collider and in fixed-target mode. Whatever the final outcome of the new extension, we can look forward to many exciting experimental results which will continue to challenge our understanding of nucleon and nuclear substructure.

\section{ACKNOWLEDGEMENTS}

This work was supported in part by the U.S. Department of Energy contract DE-AC05-84ER40150, under which the Southeastern Universities Research Association operates the Thomas Jefferson National Accelerator Facility.

\section{REFERENCES}

1. K. Hicks, hep-ph/0408001.

2. V. D. Burkert, R. De Vita and S. Niccolai, hep-ex/0408019.

3. T. Nakano et al., Phys. Rev. Lett. 91 (2003) 012002.

4. S. Stepanyan et al., Phys. Rev. Lett. 91 (2003) 252001.

5. V. Kubarovsky et al., Phys. Rev. Lett. 92 (2004) 032001.

6. C. Alt et al., Phys. Rev. Lett. 92 (2004) 042003.

7. R. A. Arndt et al., Phys. Rev. C68 (2003) 042201(R); A. Sibirtsev et al., hepph/0405099; W. R. Gibbs, nucl-th/0405024.

8. A. Dzierba et al., Phys. Rev. D69 (2004) 051901.

9. D. Weygand, talk at the X. International Conference On Hadron Spectroscopy, Aschaffenburg, Germany, 2003.

10. J. Arrington, Phys. Rev. C68 (2003) 034325.

11. J. Arrington, nucl-ex/0312017.
12. M. E. Christy et al., nucl-ex/0401030.

13. M. K. Jones et al., Phys. Rev. Lett. 84 (2000) 1398; O. Gayou et al., Phys. Rev. Lett. 88 (2002) 092301.

14. P. G. Blunden, W. Melnitchouk and J. A. Tjon, Phys. Rev. Lett. 91 (2003) 142304.

15. P. A. M. Guichon and M. Vanderhaeghen, Phys. Rev. Lett. 91 (2003) 142303.

16. Y. C. Chen et al., hep-ph/0403058.

17. J. Arrington, Phys. Rev. C69 (2004) 022201.

18. L. W. Mo and Y. S. Tsai, Rev. Mod. Phys. 41 (1969) 205.

19. L. C. Maximon and J. A. Tjon, Phys. Rev. C 62 (2000) 054320.

20. JLab experiment E04-116, W. Brooks spokesperson.

21. F. E. Close and A. W. Thomas, Phys. Lett. B212 (1988) 227.

22. G. R. Farrar and D. R. Jackson, Phys. Rev. Lett. 35 (1975) 1416.

23. X. Zheng et al., Phys. Rev. Lett. 92 (2004) 012004 .

24. I. Niculescu et al., Phys. Rev. Lett. 85 (2000) 1186 .

25. E. D. Bloom and F. J. Gilman, Phys. Rev. Lett. 16 (1970) 1140.

26. Z. E. Meziani et al., hep-ph/0404066.

27. M. Osipenko et al., hep-ph/0404195.

28. X. Ji and W. Melnitchouk, Phys. Rev. D56 (1997) 1.

29. L. S. Cardman et al., The Science Driving the 12 GeV Upgrade of CEBAF (Jefferson Lab, Newport News, Virginia, 2001).

30. L. Merminga and S. Derbenev, http://casa.jlab.org/research/elic/elic.shtml 